\title{
PharmaNews
}

Breast Care 5 | 6 | 10

Onkologische Supportivtherapie profitiert von neuen Entwicklungen

«Eigentlich sollen supportive Maßnahmen den Patienten wie ein Regenschirm schützen, aber oftmals bleibt es beim eigentlich», sagte Professor Petra Feyer, Berlin, Vorsitzende der Arbeitsgemeinschaft Supportive Maßnahmen in der Onkologie, Rehabilitation und Sozialmedizin (ASORS) der Deutschen Krebsgesellschaft, auf dem 16. Münchner Fachpresse-Workshop Supportivtherapie. Daher sind noch mehr Anstrengungen nötig, die vorhandenen Möglichkeiten in der Praxis umzusetzen. Auf Grund der längeren Überlebenszeiten und des zunehmenden Alters der Patienten sowie durch die moderne Tumortherapie selbst muss laut Professor Ingo Diel, Mannheim, in der Onkologie zunehmend mit ossären Komplikationen gerechnet werden. Die Entschlüsselung molekularer Muster hat aber auch in der Osteoonkologie neue Ansätze zugelassen. Durch die Hemmung des RANKLiganden mit Denosumab (Prolia ${ }^{\circledR}$ ) kann der
Tumor- und Therapie-bedingten Knochenzerstörung effektiv und schnell entgegengewirkt werden. Auch die antiemetische Prophylaxe wird kontinuierlich optimiert, erklärte Dr. Reinhard Musch, Berlin. In den aktualisierten Leitlinien von MASCC und ESMO vom April 2010 wird der moderne 5-HT3-Rezeptorantagonist Palonosetron $\left(\right.$ Aloxi $\left.^{\circledR}\right)$ in Kombination mit Dexamethason gegen Übelkeit und Erbrechen bei moderat emetogener Chemotherapie (ohne AC) empfohlen. Diese Substanz gibt es nun auch als orale Formulierung, die nicht nur ebenso effektiv ist wie die i.v. Form, sondern ebenfalls die Patientenzufriedenheit erhöht [1]. Für die immer noch schwierig zu behandelnde orale Mukositis zeigte das bioadhärente Mundgel Gelclair ${ }^{\circledR}$ eine gute Schmerzlinderung und erleichterte die orale Nahrungsaufnahme, fügte Dr. Hans-Peter Lipp, Tübingen, an. Ein weiteres Thema des Workshops war die Immunthrombozytopenie (ITP), eine seltene Autoimmunerkrankung des blutbildenden Systems, die sich gegen die körpereigenen Thrombozyten richtet. Der Thrombopoetin-Rezeptoragonist Romiplostim (Nplate ${ }^{\circledR}$ ) stellt laut PD Dr. Aristoteles Giagounidis, Duisburg, die erste Substanz dar, die in einer kontrollierten Studie mit bisherigen Standardtherapien bei ITP verglichen wurde und diesen überlegen war [2]

Bettina Reich, Hamburg

Quelle

16. Münchener Fachpresse Workshop am 25. November 2010

Referenzen

1 Grunberg S. et al. Eur J Cancer Suppl.: 2007;5(4)56 (abstr 1143)

2 Kuter D: N Engl J Med 2010;363:1889-1899.

Weitere Informationen bei

POMME-med GmbH

Dr. rer. nat. Petra Ortner

Von-Erckert-Straße 48, 81827 München

Tel. +49 89 454566-24, Fax -27

ortner@pomme-med.de
IVEMEND ${ }^{\circledR} 150$ mg (Fosaprepitant) als Einmalgabe jetzt in Deutschland erhältlich

Mit IVEMEND ${ }^{\circledR} 150 \mathrm{mg}$ steht seit Anfang Dezember 2010 ein neues Therapieschema von Fosaprepitant als anwendungsfreundliche Einmalgabe zur Verfügung. Fosaprepitant wird intravenös als Teil einer Kombinationstherapie zur Prävention akuter und verzögerter Übelkeit und Erbrechen bei hoch emetogener auf Cisplatin basierender Chemotherapie (HEC) sowie bei moderat emetogener Chemotherapie (MEC) eingesetzt.

Bisher wurde Fosaprepitant in einer Dosierung von $115 \mathrm{mg}$ an Tag 1 der Chemotherapie i.v. eingesetzt, gefolgt von je einer 80-mg-Kapsel
EMEND $^{\circledR}$ (Aprepitant) an den Tagen 2 und 3. Bei der Verabreichung von Fosaprepitant $150 \mathrm{mg}$ an Tag 1 der Chemotherapie ist keine weitere orale Gabe von Aprepitant erforderlich.

Basis für die Zulassung von IVEMEND ${ }^{\circledR} 150 \mathrm{mg}$ waren die Ergebnisse einer randomisierten, doppelblinden Studie an 2247 Patienten, die eine vergleichbare Wirksamkeit der einmaligen i.v.-Gabe von IVEMEND ${ }^{\circledR} 150 \mathrm{mg}$ mit der 3maligen oralen Gabe von EMEND $^{\circledR}$ zeigte. Primärer Endpunkt war ein komplettes Ansprechen während der gesamten Phase (kein Erbrechen und keine Notfallmaßnahmen bis $120 \mathrm{~h}$ nach Einleitung einer Cisplatin-basierten Therapie). Während der gesamten Phase sprachen 71,9\% der Patienten, die Fosaprepitant $150 \mathrm{mg}$ erhielten im Vergleich zu 72,3\% der
Patienten, die 3 orale Dosen Aprepitant erhielten, vollständig auf die Behandlung an. In der verzögerten Phase sprachen 74,3\% der mit Fosaprepitant $150 \mathrm{mg}$ behandelten Patienten im Vergleich zu 74,2 \% der Patienten, die das orale Aprepitant-Regime erhielten, vollständig auf die Behandlung an.

Fosaprepitant $150 \mathrm{mg}$ war vergleichbar gut verträglich wie das orale Aprepitant-Regime.

Weitere Informationen bei MSD SHARP \& DOHME GMBH

Dr. Michael Korbonits

Lindenplatz 1, 85540 Haar

Tel. +49 089 4561-1723, Fax -1329

michael.korbonits@msd.de

\section{PharmaTicker+++ PharmaTicker+++ PharmaTicker+++ PharmaTicker+++}

Boehringer Ingelheim. Mit «LUX-Breast 1» startet das Unternehmen eine klinische PhaseIII-Studie zur Prüfung von Afatinib zur Behandlung von Patientinnen mit fortgeschrittenem (metastasiertem) Mammakarzinom. Der orale Wirkstoff zählt zur neuen Generation zielgerichteter Therapien und hemmt irreversibel sowohl EGFR/HER1 (Epidermal Growth Factor Receptor / Human Epithelial Receptor 1) als auch HER2 (Human Epidermal Growth Factor Receptor 2), die beim Tumorwachstum und der Metastasierung beteiligt sind.

Boehringer Ingelheim $\mathrm{GmbH}$

Corporate Communications, Dr. Christina Janista press@ boehringer-ingelheim.de
Janssen-Cilag. Auf einem Symposium anlässlich des Hauptstadtkongresses der DGAI erläuterte Dr. Thorsten Kamp, Facharzt für Internistische Onkologie und Palliativmedizin, Wendlingen, warum Hydromorphon in der 24-Stunden-Galenik (Jurnista ${ }^{\circledR}$ ) das ideale Präparat für die Therapie tumorbedingter Schmerzen ist: Durch die stark schmerzstillenden Eigenschaften des Wirkstoffs in Kombination mit der verlässlichen Retardierung von bis zu 24 Stunden ist es möglich, eine konstante Schmerzlinderung zu erreichen.

Janssen-Cilag $\mathrm{GmbH}$

Marcus Stüttgen mstuettg@its.jnj.com
Carl Zeiss Meditec. Die neue klinische Anwendung mit INTRABEAM ${ }^{\circledR}$ eröffnet Möglichkeiten bei der Behandlung von Wirbelsäulen-Metastasen. Jetzt wurde Carl Zeiss Meditec für das gemeinsam mit der Universitätsmedizin Mannheim entwickelte Verfahren mit dem renommierten 2010 «Spine Technology Award» ausgezeichnet. INTRABEAM wird heute bereits erfolgreich in der klinischen Routine bei der intraoperativen Behandlung des Mammakarzinoms nach brusterhaltender Operation weltweit an über 100 Zentren eingesetzt.

Carl Zeiss Meditec AG

Eva Sesselmann

press@meditec.zeiss.com

\section{KARGER () 2010 S. Karger GmbH, Freiburg}

Fax +497614520714 E-mail Information@Karger.de www.karger.com 